\title{
Ion release from a composite resin after exposure to different $10 \%$ carbamide peroxide bleaching agents
}

\author{
Renata Plá Rizzolo BUENO1, Paloma Salomone VIARO'1, Paulo Cícero NASCIMENTO², Roselaine Terezinha \\ POZZOBON ${ }^{3}$
}

\begin{abstract}
1- DDS, MSc, Post Graduate Program in Dental Sciences, Federal University of Santa Maria, Santa Maria, RS, Brazil.
2- DDS, PhD, Associate Professor, Chemistry course, Post Graduate Program, Chemistry Department, Federal University of Santa Maria, Santa Maria, RS, Brazil. 3- DDS, PhD, Associate Professor, Restorative Dentistry Department, Post Graduate Program in Dental Sciences, Federal University of Santa Maria, Santa Maria, RS, Brazil.
\end{abstract}

Corresponding address: Roselaine Terezinha Pozzobon - Avenida Fernando Ferrari - 1070 - apto. 301 - Bairro: Nossa Senhora de Lourdes - Santa Maria - RS - 97050-800 - Phone: (55) 32217497 - (55) 99397722 - e-mail: rpozzobon@uol.com.br

Received: September 22, 2010 - Modification: July 14, 2011 - Accepted: July 14, 2011

\section{ABSTRACT}

\begin{abstract}
$\mathrm{O}$ bjective: This in vitro study evaluated the influence of two $10 \%$ carbamide peroxide bleaching agents - a commercial product (Opalescence PF; Ultradent Products, Inc.) and a bleaching agent prepared in a compounding pharmacy - on the chemical degradation of a light-activated composite resin by determining its release of ions before and after exposure to the agents. Material and Methods: Thirty composite resin (Filtek Z250; 3M/ ESPE) samples were divided into three groups: group I (exposed to Opalescence PF commercial bleaching agent), group II (exposed to a compounded bleaching agent) and group III (control - Milli-Q water). After 14 days of exposure, with a protocol of $8 \mathrm{~h}$ of daily exposure to the bleaching agents and $16 \mathrm{~h}$ of immersion in Milli-Q water, the analysis of ion release was carried out using a HP 8453 spectrophotometer. The values were analyzed statistically by ANOVA, Tukey's test and the paired t-tests. The significance level was set at $5 \%$. Results: After 14 days of the experiment, statistically significant difference was found between group II and groups I and III, with greater ion release from the composite resin in group II. Conclusions: The compounded bleaching agent had a more aggressive effect on the composite resin after 14 days of exposure than the commercial product and the control (no bleaching).
\end{abstract}

Key words: Composite resins. Peroxides. Dental materials.

\section{INTRODUCTION}

In recent years, with the introduction of new technologies, dentistry has progressed remarkably, developing and improving new materials. It has reached a stage where it is possible to harmonize the color and reproduce the characteristics of natural teeth, which gives everybody the opportunity to have a beautiful smile. Due to these advances, new techniques and materials have been developed for aesthetic treatment. A wide range of composite resins is now available, which enables the choice for the material that is best suited to each situation, and the correct use of either type of material is indicative of successful treatment. In addition to composite resin restorations, another aesthetic treatment option that has developed greatly is dental bleaching. There are different bleaching techniques performed using different protocols. Some are performed in the dental office (in-office bleaching), others are performed by the patient under the dentist's supervision (home bleaching), and others are associations of both that are held part in office and part at home. The bleaching agents are peroxides, which basically can be hydrogen or carbamide at different concentrations, the latter being used more frequently. With all these resources and techniques, the dentist should also, in addition to mastering the use of restorative materials, be aware of the interactions of composite resins and bleaching agents. 
Lima, et al. ${ }^{12}$ (2008), when assessing the tensile strength of the composite resin Filtek Z250 after exposure to the bleaching agents Whiteness HP (35\% hydrogen peroxide) and Whiteness Perfect ( $16 \%$ carbamide peroxide), concluded that the whitening solutions did not affect the composite resin's tensile strength. Cullen, Nelson and Sandrik ${ }^{7}$ (1993) also concluded that peroxide bleaching did not affect this property. In the same year, Bailey and Swfit Jr2 (1992) assessed the effects of bleaching agents on composite resins; by using scanning electron microscopy, they found that there were cracks on the surfaces of the resins exposed to bleaching agents. Surface roughness was assessed by Langsten, et al. ${ }^{11}$ (2002) who examined this characteristic in the resins Prodigy and Silux Plus before and after being exposed to different concentrations of carbamide peroxide (Opalescence 20\% - 3 h/day, Opalescence Quick $35 \%-1 \mathrm{~h} /$ day) for 14 days. In this study, there was no statistically significant difference between the groups. Moraes, et al. ${ }^{13}$ (2006) investigated the effect of $10 \%$ and $35 \%$ carbamide peroxide (Opalescence and Opalescence Quick) on the surface roughness of two restorative materials (Filtek - A110 and Filtek - Z250), human enamel and a feldspathic porcelain. The bleaching treatment lasted for 21 days. The $10 \%$ carbamide peroxide gel significantly increased the roughness of the porcelain group. The $35 \%$ carbamide peroxide gel increased the roughness, not only of the porcelain, but also of the resin Filtek - Z250. Polydorou, Hellwig and Auschill ${ }^{16}$ (2006) assessed the effect of home-bleaching agents on the surface texture of different restorative materials (Tetric Ceram, Tetric Flow, Enamel Plus HFO, Filtek Supreme, Definite and Vitablocs Mark II for (erec). The samples were divided into two groups (one polished with Sof-Lex sandpaper discs and the other group remained unpolished) and both were bleached with two bleaching agents (38\% Opalescence Xtra Boost and $15 \%$ Opalescence PF). The results showed that the polished samples were more stable when compared with the unpolished ones, but bleaching did not severely affect the surface texture of the studied materials. The same results were seen in the study by Bodanezi, et al. ${ }^{4}$ (2011), a clinical study, that evaluated the surface morphology of enamel and composite resin (Opallis - FGM) after bleaching for 8 days with $16 \%$ carbamide peroxide by electron microscopy, only the enamel showed morphological changes. Gurgan and Yalcin ${ }^{9}$ (2007) evaluated the effects of two bleaching agents (Crest Professional Whitestrips $-6.5 \%$ hydrogen peroxide and Vivastyle/Vivadent $-10 \%$ carbamide peroxide) in relation to surface roughness and microhardness of different restorative materials (Definite, Filtek P60 and Filtek Flow). The results showed that both bleaching agents affected the surface roughness and microhardness of all restorative materials, although the hydrogen peroxide increased the surface roughness more than the carbamide peroxide. Okte, et al. ${ }^{14}$ (2006) also evaluated microhardness after immersing the samples in pigmented agents (wine and coffee) and then bleaching them with the gels Crest Night Effect, Colgate Simply White Night and Opalescence Quick. It was observed that the microhardness decreased after immersion in bleaching agent, but with no significant difference between agents. Yu, et al. ${ }^{18}$ (2008) also reached this conclusion after assessing the composite resins Filtek - Z350 and Filtek - P60 were exposed to bleaching with $15 \%$ carbamide peroxide for 28 consecutive days. In 2007, Hannig, et al. ${ }^{10}$ assessed the effect of different bleaching techniques on the microhardness of composite resins and polyacidmodified composite resins (Tetric Flow, Tetric Evoceram and Compoglas). The bleaching agents used were: Vivastyle - 10\% carbamide peroxide ( $1 \mathrm{~h} /$ day), Whitestrips $-6.5 \%$ hydrogen peroxide (30 min/day), sodium perborate mixed with water (once every $72 \mathrm{~h}$ ), Simply White $-5.9 \%$ hydrogen peroxide (1 h/day) and Opalescence XtraBoost - 38\% hydrogen peroxide (first and fifth day for $15 \mathrm{~min}$ ). It was concluded that the agents used reduced the microhardness. The articles of Okte, et al. ${ }^{14}$ (2006), Yu, et al. ${ }^{18}$ (2008) and Hannig, et al. ${ }^{10}$ (2007) contradict the findings of Costa, et al. ${ }^{6}$ (2009), who compared four different techniques of tooth bleaching ( $7 \%$ hydrogen peroxide for $4 \mathrm{~h}$ daily for two weeks, $35 \%$ hydrogen peroxide for $30 \mathrm{~min}$, $10 \%$ carbamide peroxide for $4 \mathrm{~h}$ for 2 weeks and $35 \%$ carbamide peroxide for $30 \mathrm{~min}$ ) with controls where samples of Filtek Supreme XT (3M/ESPE) were maintained in artificial saliva for 3 weeks. No significant change in microhardness was observed.

The fracture resistance of four nanoparticle composite resins (Filtek Supreme Plus, Tetric EvoCeram, Premise and Esthet-X) after exposure to four different concentrations of bleaching gels (Opalescence PF at 10\%, 20\%, 35\% and $45 \%$ ) was tested by Cho, et al. ${ }^{5}$ (2009). Those authors found that bleaching had a significant effect on increasing the values of fracture resistance for the resin Filtek Supreme Plus, but not for the other resins. El-Murr, Ruel and St-Georges ${ }^{8}$ (2011) conducted a review on the effects of external bleaching on restorative materials and found that when it comes to composite resin, several studies have shown that bleaching agents have the potential to alter the physical properties of the restorations, but not yet demonstrated the clinical relevance that these changes may have.

Taking into account that a common clinical situation is the presence of composite resin restorations in teeth that are exposed to the action 
of bleaching agents, this study is justified and had the aim of investigating the effects of carbamide peroxide bleaching agents on resin composition by the analysis of ion release. The possible hypotheses were the null hypothesis that the bleaching agents do not affect the chemical integrity of the evaluated composite resin, and the alternative hypothesis that the bleaching agents cause the release of ions from the evaluated resin and thus lead to its chemical degradation.

\section{MATERIAL AND METHODS}

In order to carry out this study, samples ( $4 \mathrm{~mm}$ in diameter and $2 \mathrm{~mm}$ thick) of the microhybrid composite resin Filtek - Z250 (3M ESPE, St. Paul, MN, USA) were prepared using a split acrylic matrix. Two 10\% carbamide peroxide bleaching agents were used: a commercial product (Opalescence; Ultradent Products Inc., South Jordan, Utah, USA) and a compounded bleaching agent obtained from a compounding pharmacy (Dermapele, Santa Maria, RS, Brazil) (Figure 1). The immersion medium used was Milli-Q water. After preparation, the samples were stored in glass jars individually numbered and containing Milli-Q water for an initial period of 7 days and then divided into three groups $(n=10)$ : group I: exposed to the commercial bleaching agent, group II: exposed to the compounded bleaching agent, and group III (control, immersed in Milli-Q water without exposure to bleaching agents). After the initial 7 days in Milli-Q water, the samples of groups I and II were exposed to the commercial and compounded bleaching agents, respectively, in the same manner. One drop of bleaching agent was delivered in each one of 10 numbered spots marked on a large Petri dish and one composite resin sample was placed on top of each drop. The samples were covered with gauze slightly moistened with Milli-Q water and the Petri dish was capped in order to keep the samples moist, preventing dehydration and surface alterations, when placed in an oven at $37^{\circ} \mathrm{C}$. After $8 \mathrm{~h}$ of daily treatment, the samples were cleaned with a soft brush and water to remove the bleaching agents and were stored in their respective jars containing Milli-Q water at $37^{\circ} \mathrm{C}$ for $16 \mathrm{~h}$. This protocol was repeated daily during 14 days.

Analysis of ion release from resin was carried out by spectrophotometry before and after 14 days of exposure to the agents. Water was collected from the jars containing the samples and taken for analysis in the HP 8453 spectrophotometer diode array. The nominal values of ion release were recorded on spreadsheets and analyzed using SPSS (Statistical Package for Social Sciences, version 18.0; Chicago, IL, USA). Normality of distribution was verified with the Shapiro-Wilk and Levene's Test for homoscedasticity. Data regarding ion release were ranked and compared between the 3 groups by ANOVA and Tukey's post-hoc test. The release of ions in each group was compared between the two experimental periods (baseline and after 14 days) using paired t-tests. A significance level of $5 \%$ was adopted.

\section{RESULTS}

Table 1 shows the values obtained after statistical analysis of the groups at two experimental periods, i.e. before (baseline) and after 14 days of exposure of the composite to different bleaching agents. It can be observed that the ion release from composite resin did not differ among the three treatments at baseline. However, when analyzed after 14 days of exposure, there was a statistically significant difference between the group exposed to the compounded bleaching agent (group II) and group III (control) and the group exposed to the commercial bleaching agent (group I). After 14 days, the group exposed to the compounded bleaching agent presented a significantly greater ion release from composite resin $(0.023)$ than the control group (0.005) and the group exposed to Opalescence (0.010). The release of ions from the composite resin in the control group was significantly higher at the baseline $(0.007)$ than at 14 days $(0.005)$, which means that it decreased over time. In the group exposed to Opalescence, ion release increased over time, but this was not statistically significant. However, the release of ions from the composite resin exposed to bleaching agent prepared at the compounding pharmacy increased significantly comparing baseline values (0.001) and 14-day (0.023) values.

\begin{tabular}{|c|c|c|}
\hline Bleaching agent & Composition & Manufacturer \\
\hline Opalescence PF & $\begin{array}{c}10 \% \text { of carbamide peroxide, } 0.5 \% \text { of } \\
\text { potassium nitrate and } 0.11 \%(1100 \\
\text { ppm) of fluoride ion }\end{array}$ & Ultradent Products, Co \\
\hline $10 \%$ carbamide peroxide compounded & $\begin{array}{c}10 \% \text { of carbamide peroxide, stabilizers } \\
\text { and thickeners }\end{array}$ & Dermapelle Compounding Pharmacy \\
\hline
\end{tabular}

Figure 1- Bleaching agent, composition and manufacturer 
Table 1- Analyses of the values of ion release from the composite resin according to the exposure time

\begin{tabular}{lccc}
\hline & $\begin{array}{c}\text { Commercial bleaching agent } \\
\text { (Opalescence) }\end{array}$ & Compounded bleaching agent & Control (Milli-Q water) \\
\hline Baseline & $0.007(0.014)^{\mathrm{aA}}$ & $0.003(0.006)^{\mathrm{aA}}$ & $0.001(0.003)^{\mathrm{aC}}$ \\
14 days & $0.005(0.005)^{\mathrm{bB}}$ & $0.010(0.011)^{\mathrm{bA}}$ & $0.023(0.009)^{\mathrm{aA}}$ \\
\hline
\end{tabular}

For each row, lower case letters indicate a significant diference between treatments within the same experimental period For each column, different capital letters indicate a significant diference between the experimental periods while maintaining yhe same treatment

\section{DISCUSSION}

Ion release at baseline, where no sample had been exposed to bleaching agents, did not have any statistically significant difference among the three groups. After 14 days, the samples belonging to the non-bleached group (control) presented a statistically significant decrease in ion release values. This can be explained by the fact that the polymerization reaction of composite resins continues after the light source is removed. Blankenau, et al. ${ }^{3}$ (1983), Tanaka, et al. ${ }^{17}$ (1991) also showed that residual monomers of Bis-GMA and TEGDMA remain attached to the matrix, even after 7 days of immersion of the composite resin in water at $37^{\circ} \mathrm{C}$. During this period it is possible to observe a certain amount of accumulation of inorganic portion of displaced material. Anusavice ${ }^{1}$ (2003) stated that a composite resin restoration that has just been cured and polished can still have more than $50 \%$ of unreacted methacrylate groups, which may be released from the material. This fact is in agreement with Oysaed and Ruyter ${ }^{15}$ (1986) who argued that during the first 3 months after curing, the solubilization of a composite resin is more intense, decreasing gradually over time.

After 14 days of exposure to the bleaching treatments, the group in which an compounded agent was used had a statistically significant increase in the amount of ion release compared to baseline, and the group exposed to the commercial bleaching agent (Opalescence) had an increase, but it was not considered statistically significant. Although no other studies assessing ion release from composite resins exposed to bleaching agents were found, the results can be justified by the characteristics inherent to the chemical formulation of whitening agents themselves. Although the chemical carbamide peroxide is the same in both bleaching agents, the characteristics of each formulation and the more accurate quality control in manufacturing the commercial product (Opalescence) makes it safer or less aggressive, while the bleaching agent prepared at the compounding pharmacy had more chance of variations related to the production process, resulting in deleterious effects of various magnitudes.

A study by Bailey and Swift Junior ${ }^{2}$ (1992) showed that the action of bleaching agents produced cracks in the surface of the composite resins. Moraes, et al. ${ }^{13}$ (2006), Polydorou, Hellwig and Auschill ${ }^{16}$ (2006) and Gurgan and Yalcin ${ }^{9}$ (2007) showed that the resins are degraded when exposed to $10 \%$ carbamide peroxide bleaching agents, which can be evidenced by the increased surface roughness of resin composites when exposed to bleaching treatments. The study by Langsten, et al. ${ }^{11}$ (2002) indicated that the surface roughness remained constant before and after bleaching with hydrogen peroxide. Several authors ${ }^{9,10,14,18}$ evaluated the microhardness of composite resins exposed to bleaching agents and found statistically significant decreases in relation to this property. However, Costa, et al. ${ }^{6}$ (2009) did not achieve the same result, as the bleaching treatments did not alter the microhardness of the direct restorative material. Cho, et al. ${ }^{5}$ (2009) found that the values of fracture resistance of various composites increased after exposure to different concentrations of a bleaching agent. This shows that so far there is no consensus about whether there are deleterious effects of carbamide peroxide bleaching agents on composite resins but there is a strong trend indicating that they are not harmless. This study found that after 14 days of exposure of the composite resin tested led to the intensification of ion release of this material, which can accelerate chemical degradation. The clinical relevance of the results of this study is the fact that, as chemicals, both bleaching agents showed effects related to chemical degradation of the composite resin, which, when added to other chemical and physical exposures, can often lead to the premature failure of composite resin aesthetics. This leads the authors to suggest avoidance of excessive and indiscriminate exposure of composite resins restorations to $10 \%$ carbamide peroxide bleaching agents, especially when the origin and manufacturing process are not strictly controlled. Further studies should be carried out to assess the impact that these findings may have on the long-term performance of these materials considering that resins are widely used in adhesive restorative dentistry. 


\section{CONCLUSION}

From the results of this in vitro study, it was concluded that:

After 14 days of treatment, a statistically significant increase occurred in the amount of ions released from the composite resin exposed to the compounded bleaching agent compared with the control and the commercial product, which demonstrates its more aggressive effect on the restorative material.

The result leads us to reject the null hypothesis because the bleaching agents affected the chemical integrity of the composite and leads us to partially confirm the alternative hypothesis because significant difference was observed only for the compounded bleaching agent.

\section{REFERENCES}

1- Anusavice KJ. Phillips' science of dental materials. Philadelphia: W. B. Saunders; 2003.

2- Bailey SJ, Swift EJ Jr. Effects of home bleaching products on composite resins. Quintessence Int. 1992;23(7):489-94.

3- Blankenau RJ, Kelsey WP, Cavel WT, Blankenau P. Wavelength and intensity of seven systems for visible light-curing composite resins: a comparison study. J Am Dent Assoc. 1983;106(4):471-4. 4- Bodanezi A, Bittencourt ME, Bodanezi RV, Zottis T, Munhoz EA, Carlini-Júnior B. Surface modifications on aesthetically restored teeth following home bleaching with $16 \%$ peroxide carbamide. Eur J Dent. 2011;5:157-62.

5- Cho SD, Bulpakdi P, Matis BA, Platt JA. Effect of bleaching on fracture toughness of resin composites. Oper Dent. 2009;34(6):703-8.

6- Costa SXS, Becker AB, Rastelli ANS, Loffredo LCM, Andrade MF, Bagnato VS. Effect of four bleaching regimens on color changes and microhardness of dental nanofilled composite. Int J Dent. 2009;2009:313845.
7- Cullen DR, Nelson JA, Sandrik JL. Peroxide bleaches: effect on tensile strength of composite resins. J. Prosthet. Dent. 1993;69(3):247-9.

8- El-Murr J, Ruel D, St-Georges AJ. Effects of external bleaching on restorative materials: a review. J Can Dent Assoc. 2011;71:b59. 9- Gurgan S, Yalcin F. The effect of 2 different bleaching regimens on the surface roughness and hardness of tooth-colored restorative materials. Quintessence Int. 2007;38(2):e83-7.

10- Hannig C, Duong S, Becker K, Brunner E, Kahler E, Attin T. Effect of bleaching on subsurface micro-hardness of composite and a polyacid modified composite. Dental Materials. 2007;23(2):198203.

11- Langsten RE, Dunn WJ, Hartup GR, Murchison DF. Higherconcentration carbamide peroxide effects on surface roughness of composites. J Esthet Restor Dent. 2002;14:92-6.

12- Lima DA, Alexandre RS, Martins AC, Aguiar FH, Ambrosano GM, Lovadino JR. Effect of curing lights and bleaching agents on physical properties of a hybrid composite resin. J Esthet Restor Dent. 2008;20:266-75.

13- Moraes RR, Marimon JLM, Schneider LF, Correr-Sobrinho L, Camacho GB, Bueno M. Carbamide peroxide bleaching agents: effects on surface roughness of enamel, composite and porcelain. Clin Oral Invest. 2006;10:23-8.

14- Okte Z, Villalta P, Garcia-Godoy F, Lu W, Powers JM. Surface hardness of resin composites after staining and bleaching. Operative Dentistry. 2006;31(5):623-8.

15- Oysaed H, Ruyter IE. Water sorption and filler characteristics of composite for use in posterior teeth. J. Dent. Res. 1986;65(11):1315-8.

16- Polydorou O, Hellwig E, Auschill TM. The effect of different bleaching agents on the surface texture of restorative materials. Oper Dent. 2006;31(4):473-80.

17- Tanaka K, Taira M, Shintani H, Wakasa K, Yamaki M. Residual monomer (TEGDMA and Bis-GMA) of a set visible-light-cured dental composite resin when immersed in water. J Oral Rehabil. 1991;18(4):353-62.

18- Yu H, Li Q, Hussain M, Wang Y. Effects of bleaching gels on the surface microhardness of tooth-colored restorative materials in situ. J Dent. 2008;36(4):261-7. 